\title{
Características epidemiológicas según el avance de la pandemia SARS-CoV-2 en un hospital pediátrico de alta complejidad en Argentina: estudio descriptivo
}

\section{Epidemiological characteristics according to the progress of SARS-CoV-2 pandemic in a highly complex pediatric hospital in Argentina: a descriptive study}

\author{
Daiana Ferraro', Ana Paula Arias', Guadalupe Pérez', Sandra Gómez', Verónica Deschutter', Esmeralda Highton' \\ Moira Taicz', Fernanda Trugman', Micaela Picollo', Rosa Bologna' y María Teresa Rosanova'
}

'Servicio de Control Epidemiológico e Infectología, Hospital de Pediatría S.A.M.I.C "Prof. Dr. Juan P. Garrahan", Buenos Aires, Argentina.

Sin conflictos de interés.

Este estudio no recibió financiamiento.

Recibido: 24 de abril de 2021 / Aceptado: 13 de julio de 2021

\section{Resumen}

Introducción: El conocimiento de las características clínicas y evolutivas de los niños con SARS-CoV-2 está siendo continuamente actualizado. El verdadero impacto de la enfermedad en la población pediátrica es todavía desconocido. Objetivo: Describir las características clínicas, el uso de recursos y la evolución de niños con COVID-19 en el Hospital de Pediatría Garrahan, Buenos Aires, Argentina, en las primeras 20 semanas desde la identificación del primer caso. Pacientes y Métodos: Estudio descriptivo analítico, retrospectivo. Se describen las características epidemiológicas, clínicas, evolutivas y el uso de recursos hospitalarios de pacientes $<18$ años con COVID-19 confirmado. Además, se compararon dichas características según se presentaran en las primeras 10 semanas epidemiológicas desde el primer caso de COVID-19 en el hospital o en las 10 siguientes. Resultados: n: 280. La mediana de edad fue 83 meses (RIC 33-144). Fueron hospitalizados 209 pacientes $(74,6 \%)$. La mediana de días de internación fue de 8 días (RIC 3-13). Según la clasificación de gravedad de la OMS, fueron casos leves $184(65,7 \%)$, moderados $3(1,1 \%)$, graves $16(5,7 \%)$ y 20 pacientes críticos $(7,1 \%)$. Los principales motivos de ingreso a UCI no se relacionaron con la infección por SARS-CoV-2. Al comparar las características de los pacientes en los dos períodos, en el primer período hubo más frecuencia de comorbilidades subyacentes, tratamiento inmunosupresor, la consulta fue más tardía y los pacientes tuvieron más requerimientos de internación en UCI. Fallecieron en relación con la infección dos niños $(0,7 \%)$, ambos con comorbilidades

\section{Abstract}

Background: The knowledge of the clinical and evolutionary characteristics of children with SARS-CoV-2 is continuously updated. The true impact of the disease in the pediatric population is still unknown. Aim: To describe the clinical characteristics, the use of resources and the evolution of children with COVID-19 in the Garrahan Pediatric Hospital, Buenos Aires, Argentina, in the first 20 weeks from the identification of the first case. Methods: Descriptive, analytical, retrospective study. The epidemiological, clinical, evolutionary characteristics and the use of hospital resources of patients $<18$ years with confirmed COVID- 19 are described. In addition, these characteristics were compared according to whether they occurred in the first 10 epidemiological weeks from the first case of COVID-19 in the hospital or in the following ten weeks. Results: $\mathrm{n}: 280$. The median age was 83 months (IQR 33-144). 209 patients (74.6\%) were hospitalized. The median days of hospitalization was 8 days (IQR 3-13). According to the WHO severity classification, there were 184 mild cases $(65.7 \%), 3$ moderate $(1.1 \%), 16$ severe $(5.7 \%)$ and 20 critical patients $(7.1 \%)$. The main reasons for admission to the ICU were not related to SARS-CoV-2 infection. When comparing the characteristics of the patients in the two periods, in the first period there was a higher frequency of underlying comorbidities, immunosuppressive treatment, the consultation was later and the patients had more requirements for ICU admission. Two children $(0.7 \%)$ died in relation to the infection, both with severe comorbidities and severe bacterial 
graves y coinfecciones bacterianas graves. Conclusión: En este estudio predominaron los pacientes con enfermedad de base. La forma leve de la enfermedad fue la presentación más frecuente. Al inicio de la pandemia, hubo más pacientes bajo tratamiento inmunosupresor, la consulta fue más tardía y la internación fue más frecuente, prolongada y con cuadros clínicos más graves.

Palabras clave: COVID-19; SARS-CoV-2; pediatría; epidemiología; Argentina.

\section{Introducción}

$\mathrm{E}$ 131 de diciembre de 2019, China notificó la detección de casos de una nueva infección por SARS-CoV-2 denominada enfermedad por coronavirus 19 (abreviatura en inglés COVID-19), casos que posteriormente fueron también confirmados en varios países de distintos continentes. El 11 de marzo, la enfermedad fue declarada por la Organización Mundial de la Salud (OMS) una emergencia de salud pública y a la semana epidemiológica 33 , se reportaron a nivel mundial más de 21 millones de casos confirmados y 755.000 muertes, distribuidos en 216 países de los cinco continentes. Más de la mitad de los casos se registraron en la región de las Américas ${ }^{1}$.

En Argentina, el primer caso de COVID-19 se notificó el 3 de marzo de 2020, con un aumento progresivo del número de casos confirmados. El 19 de marzo de 2020 se decretaron en este país medidas de confinamiento, que incluyeron cierre de todos los centros educativos, restricción de la circulación y cierre de comercios no esenciales. Desde una etapa inicial de contención, en que la mayoría de los casos eran importados, se pasó rápidamente a una etapa de mitigación en la que predominaron los casos autóctonos. Las medidas restrictivas se prolongaron hasta noviembre de 2020 cuando la cantidad de casos de COVID-19 disminuyó. Durante todo el año 2020 en Argentina se confirmaron 1.639.874 casos de COVID-19 y 43.493 fallecimientos ${ }^{2}$. Del total de los casos notificados, 57.052 eran pacientes con edad menor a 18 años $^{3}$.

Si bien en Argentina, el confinamiento se extendió prácticamente a todo el año 2020, las restricciones fueron más estrictas hasta la semana epidemiológica 27. Sólo se permitieron actividades esenciales, flexibilizándose a partir de esa fecha, pero manteniendo el cierre de los centros educativos y de actividades en las que se concentraran más de 10 personas ${ }^{4}$. La actualización de la situación epidemiológica, la elaboración de recomendaciones internacionales y nacionales sobre el manejo de las infecciones por SARS-CoV-2 en adultos y niños, el surgimiento de nuevos conocimientos sobre el diagnóstico y tratamiento de la infección y sus complicaciones, permitieron mejorar la identificación de los casos, su manejo y en algunas situaciones su pronóstico.

Por todo lo anterior, nos propusimos describir los cambios epidemiológicos y de la utilización de los recursos hospitalarios en los niños con diagnóstico de COVID-19 en el Hospital Nacional de Pediatría "Prof. Dr. Juan P. Garrahan", Ciudad de Buenos Aires, Argentina (de ahora en adelante Hospital de Pediatría Garrahan), y comparar las características de los pacientes en las primeras 10 semanas epidemiológicas desde la aparición del primer caso en el hospital, y en las siguientes 10 . coinfections. Conclusion: In this study, patients with underlying disease predominated. The mild form of the disease was the most frequent presentation. At the beginning of the pandemic, there were more patients under immunosuppressive treatment, the consultation was later and the hospitalization was more frequent, prolonged and with more serious clinical pictures.

Keywords: COVID-19; SARS-CoV-2; pediatrics; epidemiology, Argentina.

\section{Pacientes y Métodos}

Se realizó un estudio descriptivo analítico, retrospectivo. Se incluyeron todos los pacientes $<18$ años, con diagnóstico confirmado de infección por SARS-CoV-2 desde la semana epidemiológica 17 (19.4.2020) a la 36 inclusive (5.9.2020) en el Hospital de Pediatría Garrahan. Se excluyeron los pacientes que fueron derivados a otras instituciones. Los pacientes incluidos en este estudio son parte de la cohorte de niños con infección confirmada por SARS-CoV-2 evaluados en el Hospital de Pediatría Garrahan.

El estudio se desarrolló en un hospital que es de alta complejidad, centro de atención de tercer nivel, que cuenta con más de 600 camas de internación y cinco unidades de cuidados intensivos (UCI), donde se atienden pacientes con enfermedades complejas. Desde el inicio de la pandemia, se elaboraron múltiples protocolos de atención de los pacientes en el contexto de pandemia y de diagnóstico y tratamiento de los niños con COVID-19 confirmado 5 .

Durante el período de estudio, por normativa del hospital se realizó RPC (reacción de polimerasa en cadena) para SARS-CoV-2 a todos los niños en el momento de la admisión al hospital, previo a cirugías, radioterapia y a aquellos que cumplieran con las definiciones nacionales de caso sospechoso de infección por SARS-CoV-2. Por las características del hospital, los pacientes provenían de diferentes regiones del país, con situaciones epidemiológicas variables.

Se utilizó la definición de la OMS para la clasificación clínica y definición de síndrome inflamatorio multisistémico (SIMS) asociado a SARS-CoV-2. La utilización de recursos hospitalarios se estimó a partir de los días de internación totales, días de UCI y uso de oxígeno.

Se describen las características epidemiológicas, clínicas, evolutivas y el uso de recursos hospitalarios en pacientes con diagnóstico de COVID-19 y sus complicaciones asociadas en el período de estudio. También se compararon las características de los pacientes y su evolución según el momento de presentación de los casos (Período I: semana epidemiológica (SE) 17 a 26: 19.4.20 al 27.6.20; vs Período II: SE 27 a 36: 28.6.20 a 5.9.20).

Se utilizó test de $\chi^{2}$, Rank sum o t test según el tipo de variable y su distribución. La significancia estadística entre grupos se estimó con el valor de $p$ para cada test estadístico utilizado. Se utilizó STATA 16.

Los datos de los pacientes se procesaron respetando la máxima confidencialidad de la información. La realización de este estudio y su publicación fueron aprobados por el Comité de Investigación del Hospital de Pediatría Garrahan. 
Tabla 1. Características clínicas y epidemiológicas de los pacientes con infección por SARS-CoV-2. Hospital de Pediatría Garrahan, Buenos Aires ( $n: 280$ )

\begin{tabular}{|c|c|c|}
\hline \multicolumn{2}{|l|}{ Características } & $\begin{array}{c}n(\%) \\
(n: 280)\end{array}$ \\
\hline \multicolumn{2}{|l|}{ Edad en meses: mediana ( RIC) } & $83(33-144)$ \\
\hline \multicolumn{2}{|l|}{ Edad bajo 12 meses } & $43(15,4)$ \\
\hline \multicolumn{2}{|l|}{ Sexo masculino } & $145(51,8)$ \\
\hline \multicolumn{2}{|l|}{ Enfermedad de base } & $151(53,9)$ \\
\hline $\begin{array}{l}\text { Tipo de enfermedad de } \\
\text { base }\end{array}$ & $\begin{array}{l}\text { Oncohematológica } \\
\text { Enfermedad pulmonar obstructiva crónica } \\
\text { Enfermedad neurológica } \\
\text { Receptor de trasplante de órgano sólido }\end{array}$ & $\begin{array}{l}41(14,6) \\
14(5) \\
20(7,1) \\
10(3,6)\end{array}$ \\
\hline \multicolumn{2}{|c|}{ Bajo tratamiento inmunosupresor } & $58(20,7)$ \\
\hline \multicolumn{2}{|c|}{ Días desde el inicio de síntomas hasta la consulta mediana ( RIC) } & $3(2-5)$ \\
\hline \multicolumn{2}{|l|}{ Fiebre } & $147(52,5)$ \\
\hline \multicolumn{2}{|l|}{ Internación } & $209(74,6)$ \\
\hline \multicolumn{2}{|l|}{ Internación en UCl } & $19(6,8)$ \\
\hline \multicolumn{2}{|c|}{ En UCI relacionado a COVID-19 } & $12(4,3)$ \\
\hline \multicolumn{2}{|l|}{ Requerimiento de ARM } & $10(2,6)$ \\
\hline \multicolumn{2}{|l|}{ Requerimientos de $\mathrm{O}_{2}$} & $23(8,2)$ \\
\hline Tipo de caso & $\begin{array}{l}\text { Asintomático } \\
\text { Leve } \\
\text { Moderado } \\
\text { Grave } \\
\text { Crítico }\end{array}$ & $\begin{array}{c}57(20,4) \\
184(65,7) \\
3(1,1) \\
16(5,7) \\
20(7,1)\end{array}$ \\
\hline \multicolumn{2}{|c|}{ Días de internación totales mediana ( $\mathrm{RIC}$ ) } & $8(3-13)$ \\
\hline \multicolumn{2}{|l|}{ SIMS } & $13(4,6)$ \\
\hline \multicolumn{2}{|c|}{ Mortalidad por todas las causas a 30 días } & $6(2,1)$ \\
\hline \multicolumn{2}{|c|}{ Mortalidad relacionada a COVID } & $2(0,7)$ \\
\hline
\end{tabular}

RIC: rango intercuartilo. UCl: Unidad de Cuidados Intensivos. ARM: asistencia respiratoria mecánica. SIMS: síndrome inflamatorio multisistémico asociado a SARS-CoV-2.

\section{Resultados}

Desde la semana epidemiológica 17 hasta la 36 se incluyeron 280 pacientes que cumplieron con los criterios de inclusión. De ellos, $145(51,8 \%)$ eran varones y la mediana de edad fue 83 meses (RIC 33-144) (Tabla 1). El 15,4\% tenía menos de un año de edad. Presentaban alguna comorbilidad 153 niños (53,9\%); las más frecuentes fueron enfermedad oncohematológica (n: $41 ; 14,6 \%)$ y enfermedad neurológica ( $\mathrm{n}: 20 ; 7,1 \%$ ).

Estaban asintomáticos al momento de la RPC positiva para SARS-CoV-2 57 pacientes $(20,4 \%)$.

Entre los pacientes sintomáticos, los síntomas más frecuentes fueron: fiebre en 147 pacientes $(52,5 \%)$, cuadro de vías aéreas superiores en 79 pacientes $(28,2 \%)$ y diarrea en 73 niños $(26,1 \%)$. El resto de los síntomas se presentan en la Figura 1. La mediana de tiempo desde el inicio de síntomas hasta la consulta fue de tres días (RIC 2-5).

Entre todos los pacientes de esta serie, fueron hospitalizados 209 pacientes (74,6 \%). Según la clasificación de gravedad de la OMS, fueron casos leves 184 pacientes $(65,7 \%)$, moderados $3(1,1 \%)$, graves $16(5,7 \%)$ y 20 pacientes críticos $(7,1 \%)$. La mediana de días de internación fue de ocho días (RIC 3-13).

Requirieron internación en UCI 19 pacientes $(6,8 \%)$. Los principales motivos de ingreso a UCI no se relacionaron con la infección por SARS-CoV-2, sólo 12 pacientes $(4,3 \%)$ permanecieron allí por la infección por SARS-CoV-2.

Fallecieron en relación con la infección dos niños $(0,7 \%)$, ambos con comorbilidades graves y coinfecciones bacterianas graves (neumonía por Pseudomonas aeruginosa multirresistente y bacteriemia por Staphylococcus aureus, respectivamente).

Hubo 13 pacientes con SIMS hasta la semana epidemiológica 36, de ellos cinco (8,5\%) requirieron UCI.

Al comparar las características de los pacientes que se presentaron en las primeras $10 \mathrm{SE}$ vs. las 10 siguientes,
Figura 1. Signos y síntomas de infección por SARS-CoV-2. Hospital de Pediatría Garrahan, Buenos Aires (n: 280).

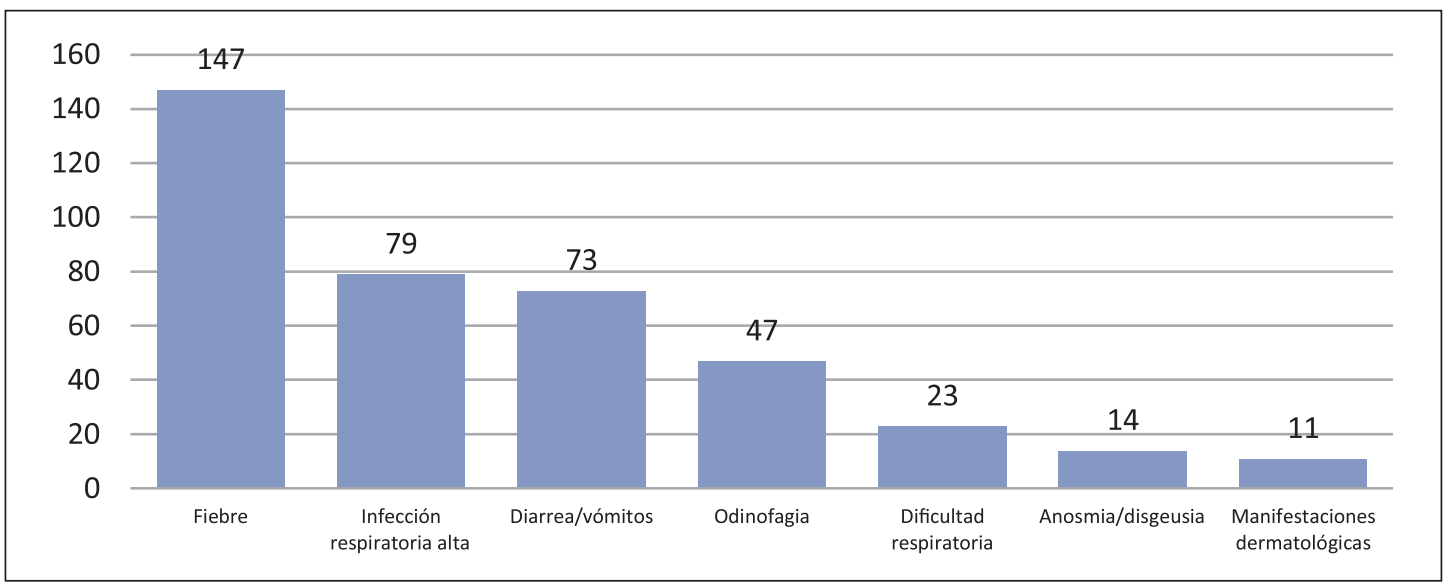


no hubo diferencias en la edad, el sexo y la frecuencia de síntomas. Sin embargo, los niños con diagnóstico de COVID-19 en el primer período tuvieron más frecuentemente comorbilidades subyacentes, tratamiento inmunosupresor y retraso en la consulta. En los pacientes que se presentaron al inicio de la pandemia, la internación fue más frecuente y prolongada (Tabla 2).

En el primer período, se realizó al menos una RPC de control para SARS-CoV-2 en 28 pacientes $(47,4 \%)$, mientras que en el segundo período a $72(32,6 \% ; p$ : 0,002).

\section{Discusión}

Al inicio de la pandemia se reportó en China una tasa de infección por SARS-CoV-2 en pacientes bajo 19 años de edad menor a $2 \%{ }^{6}$. Publicaciones posteriores de Europa, coinciden en informar que sólo 1,1-2,5\% de los infectados eran niños ${ }^{7}$. Sin embargo, en Estados Unidos de América, Blake y cols., documentaron un aumento progresivo de los casos identificados de COVID-19 en la población pediátrica: desde menos de $3 \%$ en abril de 2020 alcanzó a $12-15,9 \%$ en septiembre de $2020^{8,9}$.

En Latinoamérica los niños representan hasta la fecha alrededor de 4,2\% de los casos reportados, con diferencias sustanciales según el país ${ }^{10}$. En Argentina, la incidencia de casos en pacientes bajo 18 años fue en ascenso según avanzó la pandemia, notificándose en el reporte del Ministerio de Salud de la Nación una incidencia de 9,2\% del total de casos confirmados bajo 20 años en el año $2020^{3}$. Los primeros casos se notificaron en la semana epidemiológica 16 del año 2020, aumentando lentamente. A partir de la semana 26 se observó un aumento brusco y sostenido que se identificó como la "primer ola" epidemiológica de COVID-19.

En el Hospital de Pediatría Garrahan, el primer caso confirmado de COVID-19 se presentó en la SE 17 y luego, el número de pacientes confirmados aumentó progresivamente, siendo más numerosos a partir de la SE 27.

Dado que el Hospital de Pediatría Garrahan es un establecimiento de alta complejidad y centro de derivación, las características de los pacientes con COVID-19 confirmado en este centro difieren de lo reportado por el Ministerio de Salud para los pacientes bajo 18 años de edad. A nivel nacional, la mediana de edad de los casos pediátricos fue de 14 años (RIC 6-17) y bajo 12 meses de edad representaron el 5,3\% ${ }^{3}$. En cambio, en el estudio presente la mediana de edad es de 7 años y el 15,4\% tenía menos de 12 meses. La proporción de pacientes con comorbilidades previas difiere según las características del centro donde se realiza el estudio. En otro hospital pediátrico de la Ciudad de Buenos Aires sólo 35\% de los pacientes con COVID-19 tenían enfermedad de base, siendo el asma la más frecuente ${ }^{11}$. En el trabajo realizado en el Hospital de Pediatría Garrahan predominaron los
Tabla 2. Características clínicas y epidemiológicas de los pacientes con infección por SARS-CoV-2 comparando dos períodos de la pandemia. Hospital de Pediatría Garrahan, Buenos Aires ( $\mathrm{n:280}$ )

\begin{tabular}{|c|c|c|c|c|}
\hline \multicolumn{2}{|c|}{ Característica } & $\begin{array}{c}\text { SE } 17 \text { a } 26 \\
\text { n: } 59(21,1 \%) \\
n(\%)\end{array}$ & $\begin{array}{c}\text { SE } 27 \text { a } 36 \\
\text { n: } 221(78,9 \%) \\
n(\%)\end{array}$ & $p$ \\
\hline \multicolumn{2}{|c|}{ Edad en meses: mediana ( RIC) } & $96(51-156)$ & $82(27-139)$ & 0,16 \\
\hline \multicolumn{2}{|c|}{ Sexo masculino } & $28(47,5)$ & $117(52,9)$ & 0,56 \\
\hline \multicolumn{2}{|c|}{ Enfermedad de base } & $40(67,8)$ & $111(50,2)$ & 0,016 \\
\hline \multicolumn{2}{|c|}{ Bajo tratamiento inmunosupresor } & $18(30,5)$ & $40(18,1)$ & 0,03 \\
\hline \multicolumn{2}{|c|}{$\begin{array}{l}\text { Días desde el inicio de síntomas hasta la } \\
\text { consulta: mediana (RIC) }\end{array}$} & $5(3-9)$ & $2(1-4)$ & 0,07 \\
\hline \multicolumn{2}{|l|}{ Fiebre } & $33(55,9)$ & $114(51,6)$ & 0,55 \\
\hline \multicolumn{2}{|l|}{ Internación } & $51(86,4)$ & $158(71,4)$ & 0,019 \\
\hline \multicolumn{2}{|c|}{ Internación en UCl } & $8(13,6)$ & $11(4,9)$ & 0,02 \\
\hline \multicolumn{2}{|c|}{ En UCI relacionado a COVID } & $5(9,8)$ & $7(3,2)$ & 0,07 \\
\hline Tipo de caso & $\begin{array}{l}\text { Asintomático } \\
\text { Leve } \\
\text { Moderado } \\
\text { Grave } \\
\text { Crítico }\end{array}$ & $\begin{array}{c}9(15,2) \\
39(66,1) \\
- \\
2(3,5) \\
9(15,2)\end{array}$ & $\begin{array}{c}48(21,7) \\
145(65,6) \\
3(1,4) \\
14(6,3) \\
11(4,5)\end{array}$ & $\begin{array}{c}0,27 \\
0,94 \\
- \\
0,39 \\
0,006\end{array}$ \\
\hline \multicolumn{2}{|c|}{ Días de internación totales: mediana ( RIC) } & $9(3-24)$ & $7(2-11)$ & 0,02 \\
\hline \multicolumn{2}{|l|}{ SIMS } & $5(8,5)$ & $8(3,6)$ & 0,11 \\
\hline \multicolumn{2}{|c|}{ Mortalidad por todas las causas a 30 días } & $3(5,1)$ & $3(1,4)$ & 0,07 \\
\hline \multicolumn{2}{|c|}{ Mortalidad relacionada a COVID-19 } & $2(3,4)$ & - & - \\
\hline
\end{tabular}

SE: semana epidemiológica. RIC: rango intercuartilo. UCI: Unidad de Cuidados Intensivos. SIMS: síndrome inflamatorio multisistémico asociado a SARS-CoV-2.

pacientes con enfermedades de base y tratamiento inmunosupresor. Sin embargo, en el segundo período aumentó la proporción de niños previamente sanos con diagnóstico de infección por SARS-CoV-2, por el aumento de casos en la comunidad.

El cuadro clínico que presentan los niños con infección por SARS-CoV-2 suele ser en su mayoría leve o incluso, pueden cursar en forma asintomática ${ }^{12}$. Desde el brote de Wuhan, China, se describieron formas de presentación atípicas en la población pediátrica. La frecuencia de casos asintomáticos varía entre $20 \%{ }^{13}$ y más de $50 \%{ }^{14,15}$. En la serie presentada, a partir de RPC para SARS-CoV-2 realizadas por cribado antes de cirugías, radioterapia $\mathrm{O}$ procedimientos invasivos se identificó un $20 \%$ de niños asintomáticos.

En los niños que presentan síntomas de infección 
por SARS-CoV-2, el síntoma más frecuente reportado es fiebre, al igual que en los pacientes presentados en este estudio. También se describen diarrea, síntomas de infección respiratoria alta o manifestaciones cutáneas ${ }^{16}$.

En otras series, la frecuencia de pacientes pediátricos graves o críticos es de 6 y 4\%, respectivamente ${ }^{17}$. En nuestra casuística, más de $12 \%$ tuvo formas graves o críticas de COVID-19, siendo mayor la proporción de pacientes con formas más graves en las primeras 10 semanas. Estudios realizados en adultos, observaron también formas más graves de la enfermedad y con mayor morbimortalidad en el período inicial de la pandemia, probablemente debido a la consulta tardía, el retraso en el diagnóstico y el desconocimiento de la enfermedad ${ }^{16,18}$.

En relación al mejor conocimiento de la naturaleza de la infección, los pacientes internados en el segundo período permanecieron menos días hospitalizados que los del primer período, y esta diferencia también se observó en los niños con enfermedades subyacentes. La elaboración y difusión de guías de diagnóstico, tratamiento, y recomendaciones para el seguimiento de diferentes patologías, optimiza el uso de recursos y la utilización adecuada de las tecnologías ${ }^{17,19-21}$.

Los pacientes de esta serie, requirieron con frecuencia ingreso a UCI. Sin embargo, en la mayoría de los casos el motivo de admisión en UCI no estuvo relacionada con el COVID-19, sobre todo al inicio de la pandemia cuando la consulta por otras patologías era más tardía. En otras publicaciones también se ha destacado el aumento en la morbimortalidad relacionado con el retraso en la consulta por otras causas no relacionadas con la infección por SARS-CoV-222.

A nivel mundial, con el aumento exponencial del número de casos de COVID-19, se reportó un aumento sustancial de la demanda al sistema de salud, las internaciones generales y en $\mathrm{UCI}^{23}$. La gestión de los recursos sanitarios (camas de internación, dispositivos médicos, profesionales de la salud, camas en UCI) es fundamental para la adecuada atención de los pacientes. En este estudio se observó una disminución estadísticamente significativa en los días de internación totales, de admisión en UCI y de realización de RPC de control a partir del mejor conocimiento de la enfermedad.

En este estudio dos pacientes fallecieron en relación al COVID-19, estimando una mortalidad en toda la serie de $0,7 \%$, mientras que la mortalidad global en pacientes bajo 18 años de edad en Argentina fue de 0,09\% para el año $2020^{3}$. Esta diferencia se relaciona con el tipo de institución donde se concentran pacientes con enfermedades crónicas y graves. Ambos pacientes tenían enfermedades de base graves y fallecieron con coinfecciones bacterianas también graves.

Los niños con infecciones por SARS-CoV-2 se presentan oligosintomáticos y por ello representan un desafío diagnóstico. Además, aunque son infrecuentes las formas graves, requieren un sistema de salud preparado para la atención de estos niños.

Si bien el presente estudio describe la epidemiología y las características clínicas de los niños con SARS-CoV-2 sólo durante las primeras 20 semanas epidemiológicas del año 2020, desde la identificación del primer caso, permite observar las principales características de la enfermedad en la población pediátrica. Una de las principales fortalezas del trabajo es la documentación de todos los casos confirmados en un hospital de alta complejidad desde el inicio de la pandemia, su evolución y morbilidad asociadas. Además, la alta proporción de pacientes con comorbilidades subyacentes y COVID-19, aporta conocimiento respecto al comportamiento de SARS-CoV-2 en este tipo de niños.

\section{Conclusiones}

En este estudio descriptivo de niños con infección por SARS-CoV-2 confirmada en las primeras 20 semanas desde la identificación del primer caso en el Hospital de Pediatría Garrahan, predominaron los pacientes con enfermedad de base. Las formas asintomáticas de COVID-19 fueron frecuentes y fue mayoritaria la enfermedad leve. Al inicio de la pandemia, la consulta fue más tardía y la internación fue más frecuente, prolongada y con cuadros clínicos más graves. Fallecieron dos pacientes en relación con COVID-19, ambos con afecciones subyacentes y coinfecciones bacterianas en el primer período.

El conocimiento de la enfermedad, la elaboración y difusión de guías de diagnóstico y manejo de los pacientes con sospecha o confirmación de infección por SARSCoV-2 es fundamental para mejorar la morbimortalidad asociada a esta patología.

\section{Referencias bibliográficas}

1.- Who.int. Coronavirus disease (COVID-19) Situation Report-208 Data as received by WHO from national authorities by 10:00 CEST, 15 August 2020 [citado el 15 de marzo de 2021]. Disponible en: https://www.who.int/ docs/default-source/coronaviruse/situation- reports/20200815-covid-19-sitrep-208. pdf?sfvrsn=9dc4e959_2.

2.- 53/ Boletín Integrado de Vigilancia. EDICIÓN ESPECIAL Vigilancia de COVID-19 y Vigilancia de Arbovirosis SE 1 a 53/2020 SE 1. a. BIV Edición especial | SE 1 a 53/2020 [Internet]. Gob.ar. [citado el 15 de marzo de 2021]. Disponible en: https://bancos.salud.gob.
ar/sites/default/files/2021-02/BIV-530-1-a-53COVID Arbovirosis.pdf.

3.- Sala de Situación COVID-2019 nuevo coronavirus 2019 (información disponible al 09/01/2021 (SE 1 completa) Niñez/ Adolescencia y COVID-19. Ministerio de Salud Argentina. https://www.argentina.gob.ar/sites/ default/files/sala_pediatria_9_1_21.pdf 
4.- Aislamiento social, preventivo y obligatorio para toda la población. Ministerio de Salud de la Nación Argentina. Disponible en: https:// www.argentina.gob.ar/coronavirus/medidasgobierno

5.- Manejo de pacientes pediátricos con COVID-19. Agosto de 2020. Servicio de Epidemiología e Infectología. Hospital de Pediatría "Prof. Dr. J.P. Garrahan". https://www.garrahan.gov.ar/images/ coronavirus/Manejo\%20de \%20pacientes $\% 20$ pediatricos\%20con\%20COVID\%2019.pdf.

6.- $\quad \mathrm{Wu}$ Z, McGoogan J M. Characteristics of and important lessons from the coronavirus disease 2019 (COVID-19) outbreak in China: Summary of a report of 72314 cases from the Chinese center for disease control and prevention. JAMA. 2020; 323(13): 1239-42. doi: 10.1001/ jama.2020.2648.

7.- CDC COVID-19 Response Team. Coronavirus disease 2019 in children - United States, February 12-April 2, 2020. MMWR Morb Mortal Wkly Rep. 2020; 69(14):422-6. doi: 10.15585/mmwr.mm6914e4.

8.- Sisk B, Cull W, Harris J M, Rothenburger A, Olson L. National trends of cases of COVID-19 in children based on US State Health Department data. Pediatrics. 2020; 146(6):e2020027425. doi: 10.1542/peds.2020-027425.

9.- ECDC: European Center For Disease Prevention and Control. Gov.lv. Coronavirus disease 2019 (COVID-19) in the EU/EEA and the UK-eighth update [citado el 15 de marzo de 2021]. Disponible en: https://www.zva.gov. $\mathrm{lv} / \mathrm{sites} / \mathrm{default} /$ files/inline-files/covid-19-rapidrisk-assessment-coronavirus-disease-2019eighth-update-8-april-2020.pdf.

10.- Atamari-Anahui N, Cruz-Nina N D, Condori-Huaraka M, Núñez-Paucar H, Rondón-Abuhadba E A, Ordoñez-Linares $\mathrm{M}$ E, et al. Characterization of coronavirus disease 2019 (COVID-19) in children and adolescents in Latin American and the Caribbean countries: A descriptive study. Medwave. 2020; 20(8):e8025. doi: 10.5867/ medwave.2020.08.8025.

11.- Raiden S, Cairoli H, Potasnik J, Di Lalla S, Chiolo M J, Torres F, et al. Children hospitalized for COVID-19 during the first winter of the pandemic in Buenos Aires, Argentina [Internet]. bioRxiv. 2020 [citado el 15 de marzo de 2021]. p. 2020.11.05.20225300. Disponible en: https://www.medrxiv.org/content/10.1101/2020. 11.05.20225300v2.

12.- Who.int. Report of the WHO-China Joint Mission on Coronavirus Disease 2019 (COVID-19) [citado el 15 de marzo de 2021] Disponible en: https://www.who.int/docs/ default-source/coronaviruse/who-china-jointmission-on-covid-19-final-report.pdf

13.- Li B, Zhang S, Zhang R, Chen X, Wang Y, Zhu C. Epidemiological and clinical characteristics of COVID-19 in children: a systematic review and meta-analysis. Front Pediatr. 2020; 8. doi: 10.3389/fped.2020.591132

14.- Assaker R, Colas A-E, Julien-Marsollier F, Bruneau B, Marsac L, Greff B, et al. Presenting symptoms of COVID-19 in children: a metaanalysis of published studies. Br J Anaesth. 2020; 125(3). doi: 10.1016/j.bja.2020.05.026.

15.- Poline J, Gaschignard J, Leblanc C, Madhi F, Foucaud E, Nattes E, et al. Systematic Severe Acute Respiratory Syndrome Coronavirus 2 screening at hospital admission in children: a French prospective multicenter study. Clin Infect Dis 2021; 72(12): 2215-7. doi: 10.1093/ $\mathrm{cid} / \mathrm{ciaa} 1044$.

16.- Dennis J M, McGovern A P, Vollmer S J, Mateen B A. Improving survival of critical care patients with coronavirus disease 2019 in England: a national cohort study, March to June
2020*. Crit Care Med. 2021; 49(2): 209-14 doi: 10.1097/CCM.0000000000004747.

17.- Roland D, Harwood R, Bishop N, Hargreaves D, Patel S, Sinha I. Children's emergency presentations during the COVID-19 pandemic. Lancet Child Adolesc Heal. 2020; 4(8). e32-e33. doi: 10.1016/S23524642(20)30206-6.

18.- Asch D A, Sheils N E, Islam M N, Chen Y, Werner R M, Buresh J, et al. Variation in US hospital mortality rates for patients admitted with COVID-19 during the first 6 months of the pandemic. JAMA Intern Med. 2021; 181(4): 471-8. doi: 10.1001/jamainternmed.2020.8193.

19.- Bodington R, Bhandari S. Falling usage of hospital-based emergency care during the COVID-19 pandemic in the UK. J R Coll Physicians Edinb. 2020; 50(2): 207-14 doi: 10.4997/JRCPE.2020.234.

20.- Dellit T H, Owens R C, McGowan J E, Gerding D N, Weinstein R A, Burke J P, et al. Infectious Diseases Society of America and the Society for Healthcare Epidemiology of America Guidelines for developing an institutional program to enhance antimicrobial stewardship. Clin Infect Dis. 2007; 44(2): 1595-77. doi: 10.1086/510393.

21.- Newman R E, Hedican E B, Herigon J C, Williams D D, Williams A R, Newland J G. Impact of a guideline on management of children hospitalized with community-acquired pneumonia. Pediatrics. 2012; 129(3). e597-604. doi: 10.1542/peds.2011-1533.

22.- Masroor S. Collateral damage of COVID-19 pandemic: delayed medical care. J Card Surg. 2020; 35(6): 1345-7. doi: 10.1111/jocs.14638.

23.- Grasselli G, Pesenti A, Cecconi M. Critical care utilization for the COVID-19 outbreak in Lombardy, Italy: early experience and forecast during an emergency response. JAMA. 2020; 323(16): 1545-6. doi: 10.1001/jama.2020.4031. 